\title{
Spectroscopic and photometric study of two B-type pulsators in eclipsing systems
}

\author{
Dominik Drobek and Andrzej Pigulski
}

\author{
Instytut Astronomiczny, University of Wrocław, \\ ul. Kopernika 11, 51-622 Wrocław, Poland \\ email: drobek@astro.uni.wroc.pl
}

\begin{abstract}
Pulsating stars in eclipsing binary systems play an important role in asteroseismology. The combination of their spectroscopic and photometric orbital solutions can be used to determine, or at least to constrain, the masses and radii of components. To successfully perform any seismic modelling of a star, one has to identify at least some of the detected modes, which requires precise time-series photometric and spectroscopic observations. This work presents a progress report on the analysis of two $\beta$ Cephei-type stars in eclipsing binaries: HD 101794 (V916 Cen) and HD 167003 (V4386 Sgr).
\end{abstract}

Keywords. stars: individual: HD 101794, HD 167003, stars: oscillations, binaries: eclipsing

\section{Observations and data reduction}

HD 101794 and HD 167003 have been observed at the South African Astronomical Observatory (SAAO) between May 2 and 19, 2009. The $U B V R I$ time-series photometry has been acquired using the UCT CCD detector at the 1.0-m telescope. Spectroscopic observations were carried out using the GIRAFFE échelle spectrograph at the 1.9-m telescope. We obtained spectra in the wavelength range between $4200 \AA$ and $6900 \AA$ with a resolution $R \approx 39000$. Stellar magnitudes were calculated using the program DAOPHOT II (Stetson 1987). Spectra were wavelength-calibrated and extracted using the IRAF software package (Tody 1993). The échelle orders were merged and normalised with a program of our own, and the radial velocities were calculated by cross-correlating the observed spectra with non-LTE models of Lanz \& Hubeny (2007) using the method of Tonry \& Davis (1979).

\section{Data analysis and results}

The spectrum of HD 101794 features very broad lines, the broadening being caused by rapid rotation. This is not surprising at all, since HD 101794 is a known Be star. Unfortunately, the resulting broadening reduces the accuracy of the radial velocities obtained with cross-correlation. For this reason the analysis of HD 101794 requires more consideration. The star is still under study, and the results will not be discussed here.

The radial velocity time-series data of HD 167003 were subjected to Fourier analysis. The orbital period was estimated at $10.88 \mathrm{~d}$, which is close to the value of $10.79824 \mathrm{~d}$ obtained from the analysis of the All Sky Automated Survey phase 3 (ASAS-3) $V$ band photometry by Pigulski \& Pojmański (2008). We adopted their period value in our subsequent analysis. Apart from the effects of orbital motion, at least four frequencies arising from stellar pulsations are present in the power spectrum. The frequency $f_{1}=$ $7.351 \mathrm{~d}^{-1}$ is only seen in the radial velocity data. Our $f_{2}=6.771 \mathrm{~d}^{-1}$ and $f_{3}=7.023 \mathrm{~d}^{-1}$ correspond to $f_{1}$ and $f_{3}$ found by Pigulski \& Pojmański (2008). Our last frequency, $f_{4}=$ 
$8.451 \mathrm{~d}^{-1}$, is not seen in their photometric data, while their $f_{4}$ was not detected in our radial velocity measurements. In our newly acquired SAAO multicolour photometry, we detected frequencies corresponding to $f_{1}, f_{2}$ and $f_{3}$ found by the aforementioned authors.

Once the frequencies of pulsation were determined, their contribution was removed from the original radial velocity data. We attempted to model the radial velocity changes arising from orbital motion, and arrived at the following set of parameters: orbital period $P_{\text {orb }}=10.79824 \mathrm{~d}$ (fixed); semi-amplitude $K=(31.8 \pm 0.6) \mathrm{km} \mathrm{s}^{-1}$; eccentricity $e=$ $0.061 \pm 0.013$; argument of periastron $\omega=(299 \pm 8)^{\circ}$; systemic velocity $\gamma=(-30 \pm$ $0.4) \mathrm{km} \mathrm{s}^{-1}$ and the time of periastron passage $T_{0}=$ HJD $2454965.53 \pm 0.23$. The standard deviation from the fit amounts to $2.9 \mathrm{~km} \mathrm{~s}^{-1}$.

\section{Discussion}

The results for HD 167003 are very encouraging. First of all, we have confirmed it is indeed a pulsating star in a binary system. In addition, we have confirmed that the orbital period amounts to $10.79824 \mathrm{~d}$ and is not twice as long, as initially suspected by Pigulski \& Pojmański (2008). This star seems to be a single-lined spectroscopic binary, and our modelling suggests that the orbit is close to circular.

Pigulski \& Pojmański (2008) detected only the primary eclipse in the ASAS-3 photometry of HD 167003. Our initial hypothesis was that the lack of the secondary eclipse is caused by a highly eccentric orbit. In light of the results of our modelling, we now know this cannot be the case. This suggests that the secondary eclipse is very shallow, and that the contribution of the secondary component to the total flux is small. While our present photometry is more accurate than the ASAS-3 photometry used by the previous investigators, we are also unable to find the secondary minimum in our observations. This could be because the orbital period of HD 167003 is quite long, and our phase coverage is incomplete. However, we managed to detect hints of a minute reflection effect.

Once the orbital inclination is known from the light curve modelling, we will be able to use the mass function to constrain the masses of components. From the fact that at least two of the photometrically detected modes are also seen in the radial velocity data, it seems probable that the attempts to identify mode degrees from amplitude ratios and phase differences will be successful. Therefore, this star seems to be a very good candidate for asteroseismic analysis.

\section{Acknowledgements}

The authors would like to thank G. Kopacki for acquiring photometric observations of our targets. D. Drobek would like to thank E. Niemczura for her help with the model spectra, and Z. Kołaczkowski and J. Molenda-Żakowicz for their assistance with the IRAF software package. This work has been supported by the National Science Centre grants no. 2011/01/N/ST9/00400 and no. 2011/03/B/ST9/02667.

\section{References}

Lanz, T. \& Hubeny, I. 2007, ApJS, 169, 83

Pigulski, A. \& Pojmański, G. 2008, A\&A, 477, 917

Stetson, P. B. 1987, PASP, 99, 191

Tody, D. 1993, ASP-CS, 52, 173

Tonry, J. \& Davis, M. 1979, AJ, 84, 1511 